\title{
Non-gravitational forces acting on small bodies
}

\author{
Miroslav Brož ${ }^{1}$, D. Vokrouhlický${ }^{1}$, W.F. Bottke ${ }^{2}$, D. Nesvorný ${ }^{2}$, \\ A. Morbidelli ${ }^{3}$ and D. Čapek ${ }^{1}$ \\ ${ }^{1}$ Institute of Astronomy, Charles University, Prague, V Holešovičkách 2, 18000 Prague 8, \\ Czech Republic \\ email: vokrouhl@mbox.cesnet.cz,mira@sirrah.troja.mff.cuni.cz,capek@sirrah.troja.mff.cuni.cz \\ ${ }^{2}$ Southwest Research Institute, 1050, Walnut St., Suite 400, Boulder, CO-80302, USA \\ email: bottke@boulder.swri.edu, davidn@boulder.swri.edu \\ ${ }^{3}$ Observatoire de Nice, Dept. Cassiopee, BP 4229, 06304 Nice Cedex 4, France \\ email: morby@obs-nice.fr
}

\begin{abstract}
Non-gravitational perturbations, regardless being many orders of magnitude weaker than gravity, hold keys to fully understand the evolution of small Solar System bodies. This is because individual bodies, or their entire groups, manifest traces of a long-term accumulated changes by these effects.

For meteoroids and small asteroids in the $10 \mathrm{~cm}-10 \mathrm{~km}$ size range, the principal non-gravitational force and torque arise from an anisotropic thermal emission of the absorbed solar radiation. Related perturbations of the orbital and rotational motion are called the Yarkovsky and YORP effects.

We review the most important Yarkovsky- and YORP-driven processes, in the Main Asteroid Belt. These include: steady and size-dependent semimajor axis drift, secular changes of rotational period and obliquity, efficient transport towards low-order resonances, interaction with weaker higher-order resonances, captures in secular and spin-orbit resonances.

Many independent observations can be naturally interpreted in the framework of Yarkovsky/YORP models, like cosmic ray exposure ages of meteorites, current population and sizedistribution of near-Earth objects, the existence of unstable resonant asteroids or the structure of asteroid families.
\end{abstract}

Keywords. minor planets, asteroids; interplanetary medium; radiation mechanisms: thermal

\section{Introduction}

Current observations of small Solar System bodies provide many important constraints for dynamical studies. Laboratory analyses of collected meteorite samples, astrometric and photometric observations of small asteroids in the Earth's neighbourhood or relatively larger asteroids orbiting in the Main Asteroid Belt allowed us to recognise, during the last ten years, the importance of non-gravitational phenomena affecting their orbital evolution.

In this review, we are going to focus on small asteroidal bodies in the size-range from $10 \mathrm{~cm}$ up to $10 \mathrm{~km}$, which do not exhibit any outgassing and cometary activity. The principal accelerations affecting the motion of these small bodies are listed in Table 1.

The largest non-gravitational accelerations caused by the interaction with the solar radiation field - like the Yarkovsky/YORP effect, the radiation pressure or the PoyntingRobertson drag - are, roughly speaking, 10 orders of magnitude weaker than solar gravity. At a first glimpse, they seem to be too subtle phenomena, but we have to take into 
Table 1. The approximate values of radial and transversal accelerations affecting bodies in the size-range $10 \mathrm{~cm}$ to $10 \mathrm{~km}$. The solar gravity is scaled to unity. For comparison, typical gravitational perturbations by planets and large asteroids are $G M_{\mathrm{pl}} \simeq 10^{-3}$ and $G M_{\text {ast }} \lesssim 10^{-9}$.

\begin{tabular}{lcc}
\hline acceleration & radial & transversal \\
\hline gravity & $G M_{\odot} \simeq 1$ \\
Yarkovsky/YORP effect & $10^{-7}$ to $10^{-11}$ & $10^{-8}$ to $10^{-12}$ \\
radiation pressure & $10^{-6}$ to $10^{-11}$ & \\
Poynting-Robertson drag & \multicolumn{2}{c}{$10^{-10}$ to $10^{-15}$} \\
solar wind, Lorentz force, plasma drag & \multicolumn{2}{c}{$<10^{-15}$} \\
\hline
\end{tabular}

account also the direction of the acceleration vector and the effect of its eventual longterm accumulation.

Of course, a small radial acceleration, not exceeding the solar gravity, does not have significant orbital effects (it only slightly decreases or increases the orbital velocity), while a transversal acceleration may cause a secular change of energy (and hence the semimajor axis of the orbit). Some types of accelerations also tend to average-out along the orbit, while others can accumulate over millions or even billions of years. If we take into the account these two issues, the Yarkovsky/YORP effect is by far the strongest non-gravitational force in the size-range $10 \mathrm{~cm}$ to $10 \mathrm{~km}$ and, hereinafter, we will focus on the Yarkovsky/YORP only.

How much a body can change its orbit? What are the secular effects? Typically, the Yarkovsky/YORP force can push a 10-m meteoroid's semimajor axis by $0.1-0.2 \mathrm{AU}$, before being disrupted by a random collision with another body. Similarly, a small 1-km Main-Belt asteroid can move by 0.05 AU (within its collisional lifetime). These are certainly significant shifts, comparable to the distances between major resonances or to the sizes of asteroid families (i.e., the prominent concentrations of asteroids in the properelement space). They give a hint that the Yarkovsky/YORP effect plays an important role in the evolution of small Solar-System bodies.

We present a brief overview of Yarkovsky and YORP effects principles in Section 2 and the most direct observational evidences for these phenomena in Section 3. Section 4 is devoted to various unstable populations, which the Yarkovsky/YORP helps to sustain, and Section 5 to evolutionary processes shaping asteroid families.

\section{The Yarkovsky/YORP effect principles}

The basic principle of the Yarkovsky/YORP thermal effect is the absorption of solar radiation by a body and its anisotropic thermal reemission. The temperature differences on the surface, together with an uneven shape of the body, then lead to a recoil force and torque (Figure 1). (A detailed discussion on the mathematical theory describing the Yarkovsky/YORP effect can be found in Bottke et al. (2002) and references therein.) Contrary to the direct radiation pressure and its relativistic counterpart, the PoyntingRobertson effect, the radiation is absorbed and thermally reprocessed here. Due to a finite thermal conductivity of the material, there is some "thermal lag" between the absorption and the emission. This is also the reason, why the Yarkovsky/YORP effect sensitively depends on the rotational state (obliquity $\gamma$ and period $P$ ).

The Yarkovsky/YORP effect is negligible in case of very small and very large bodies: the upper limit for size $D$ is a natural consequence of the fact, that the force is approximately proportional to the surface area $\left(D^{2}\right)$, the mass $\propto D^{3}$ and thus the resulting acceleration $\propto 1 / D$. The lower limit is given by the conduction of heat across the whole 


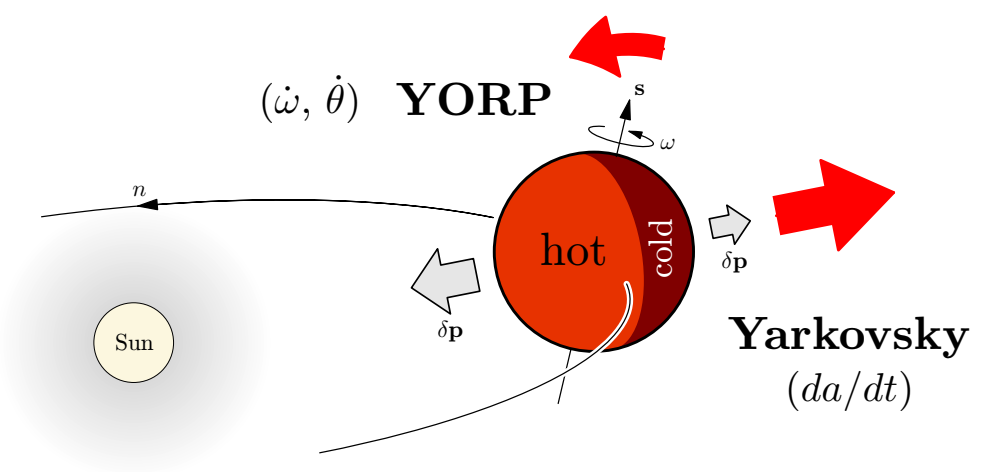

Figure 1. An illustration of the Yarkovsky/YORP effect principle. As an asteroid absorbs the solar radiation, its part facing the Sun becomes hotter than the reverse one. The infrared emission from the surface is then anisotropic, what gives rise to the Yarkovsky force, affecting the orbital motion of the asteroid, and the YORP torque, modifying the spin state.

small body, which effectively diminishes temperature differences on the surface and the corresponding infrared emission is then almost isotropic.

In the next sections, we will need to know the principal secular effects of the force and torque on the orbital and rotational dynamics. The Yarkovsky force is related to the orbital dynamics (Rubincam 1995; Vokrouhlický 1998, 1999). Its diurnal variant, driven by the rotational frequency, dominates for bodies with low thermal conductivity (e.g., with regolith on the surface). It can either increase or decrease semimajor axis $a$ and the change $\Delta a$ is proportional to the cosine of the obliquity $\gamma$. In case of the seasonal variant, the changes of temperature on the surface are mainly driven by the orbital frequency. It is a usual situation for bodies with higher thermal conductivity (regolith-free surface). The semimajor axis $a$ steadily decreases and $\Delta a \propto-\sin ^{2} \gamma$.

The YORP torque (Rubincam 2000; Vokrouhlický \& Čapek 2002) works for nonspherical bodies only. It has an asymptotic behaviour - it pushes the obliquity towards 0 or $180^{\circ}$ and the rotation period towards 0 or $\infty$. (We note, however, that the behaviour of the YORP and collisional evolution close to these asymptotic spin states is poorly understood today and it will certainly be a subject of forthcoming studies.) Because of the dependence of the Yarkovsky force on the obliquity we can expect a complicated interplay between the Yarkovsky and YORP effects.

Of course all variants of the Yarkovsky forces and the YORP torque are caused by a single temperature distribution on the surface of the body - they are actually a single phenomenon. Nevertheless, we find the above division conceptually useful.

What do we need to calculate the Yarkovsky/YORP? To properly calculate the temperature distribution on the surface of an asteroid (and then straightforwardly the corresponding IR emission, force and torque) we need to know its orbit (i.e., the position of the radiation source), size and shape, spin axis orientation and period, mass, density of surface layers, albedo, thermal conductivity, capacity and IR emissivity of the material.

These are many a priori unknown parameters. In the "worst" case (and for vast majority of asteroids), we know only the orbit and broad-band photometry results (from which we can "guess" an approximate albedo, size and thermal parameters). How to overcome this lack of physical parameters? One possibility is to study only asteroids known very well, like (6489) Golevka (Figure 2). However, we can also use a collective dynamics approach - study whole groups of bodies (like asteroid families) and treat the unknown 

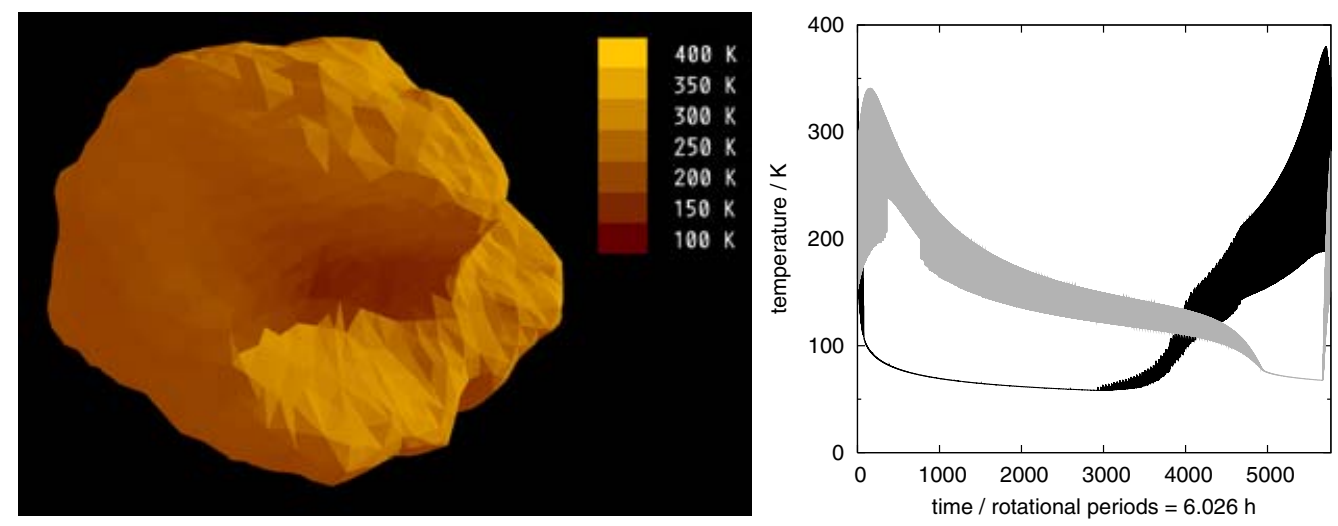

Figure 2. The temperature distribution on the surface of the asteroid (6489) Golevka, calculated by a numerical solution of the 1-dimensional heat diffusion equation, individually for all 4092 surface elements of the shape model. For two selected surface elements, located on roughly opposite sides of the body, we plot the time evolution of the temperature (the time is counted as the number of rotations and covers one complete orbit). Both seasonal and diurnal variations of the temperature, due to the changing distance from the Sun, illumination geometry and shadowing, are clearly visible. Adapted from Chesley et al. (2003).

thermal parameters as statistical quantities, it means to select a reasonable probability distribution and assign them randomly to the individual bodies.

\section{The Yarkovsky and YORP: the most direct observational evidence}

Following a previous prediction by Vokrouhlický et al. (2000), Chesley et al. (2003) were the first to directly detect the non-gravitational semimajor axis drift due to the Yarkovsky effect. Vokrouhlický et al. (2000) computed the position of (6489) Golevka during its 2003 close approach to the Earth using all previous radar and optical astrometry data and two models of Golevka's motion: (i) purely gravitational only and (ii) with the Yarkovsky acceleration included (Figure 3).

The respective radar ranging to Golevka, reported by Chesley et al. (2003), confirmed the $15 \mathrm{~km} O-C$ difference in the distance from the dish, what is outside $3-\sigma$ error interval of the purely gravitational model, but it fits very well with the Yarkovsky model. Because the latter involves a non-gravitational acceleration, they were also able to constrain the bulk density of Golevka to $2.7_{-0.6}^{+0.4} \mathrm{~g} / \mathrm{cm}^{3}$.

The current state-of-the-art model by Čapek \& Vokrouhlický (2005) assumes Golevka consists of two layers: low conductivity surface and high conductivity core. It enables to put a lower limit for the surface thermal conductivity $K$, which should be at least $10^{-2}$ or $10^{-1} \mathrm{~W} / \mathrm{m} / \mathrm{K}$, (i.e., substantially larger than the laboratory-measured conductivity of the lunar regolith $10^{-3} \mathrm{~W} / \mathrm{m} / \mathrm{K}$ ). This is in a rough agreement with thermophysical models, which Delbó et al. (2003) use to interpret observed infrared fluxes coming from Near-Earth asteroids. The average value of $K$ for all observed NEA's seems to be of the same order.

Unfortunately, we do not have any direct measurement of the YORP effect yet. However, a strong evidence of the ongoing YORP evolution comes from the analysis of a group of Koronis-family asteroids, which has a bimodal obliquity distribution (Slivan 2002; Slivan et al. 2003). The prograde group has periods $7.5-9.5 \mathrm{~h}$, obliquities $42^{\circ}-50^{\circ}$ and even similar ecliptic longitudes of the poles within $40^{\circ}$. The values for the retrograde group are $P<5 \mathrm{~h}$ or $>13 \mathrm{~h}$ and $\gamma \in\left(154^{\circ}, 169^{\circ}\right)$ (Figure 4$)$. This observational result 


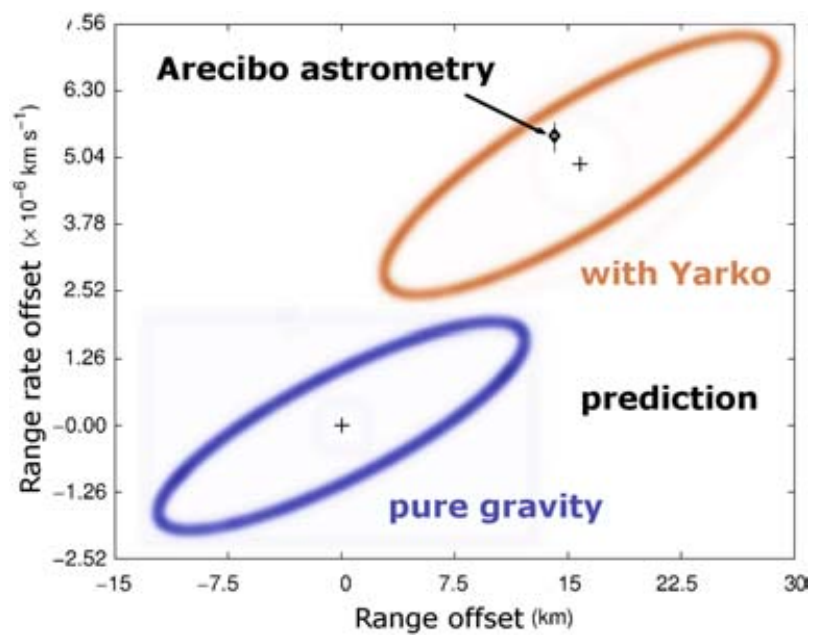

Figure 3. Range vs. range rate (i.e., the quantities measured by radar) for the close approach of (6489) Golevka in May 2003. The predictions of the two theoretical models of Golevka's motion, purely gravitational and with Yarkovsky, are plotted with their $90 \%$ confidence ellipses. The astrometric observation by the Arecibo radar is denoted by the black point and arrow. Adapted from Chesley et al. (2003).
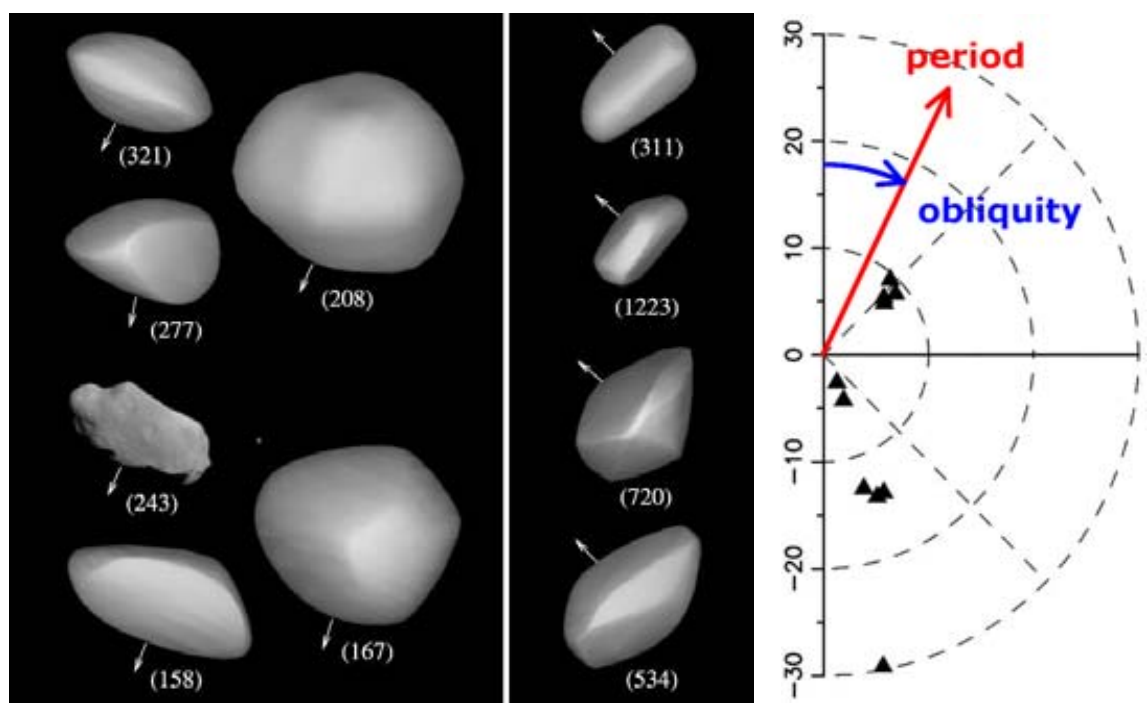

Figure 4. Shape models and spin vectors of 11 Koronis family asteroids (left) and a polar plot period vs. obliquity for the same group (right). Adapted from Slivan et al. (2003).

was very surprising, because collisions should produce a random distribution of rotational states, surely not the bimodal.

Vokrouhlický et al. (2003) thus constructed a model of spin state evolution, which included solar torques and the YORP thermal torque. Let's take the prograde-rotating asteroids as an example (Figure 5). They analysed the evolution of asteroids, which initially had periods $P=4-5 \mathrm{~h}$ and obliquities $\gamma$ evenly distributed in the interval $\left(0^{\circ}, 90^{\circ}\right)$. They found the evolution is firstly driven by the YORP effect toward an asymptotic state ( $\gamma$ decreases and $P$ increases). After some $1 \mathrm{~Gy}$, when the precession rate reaches the value $\simeq 26^{\prime \prime} / \mathrm{y}$, the spin is captured in the $s_{6}$ spin-orbit resonance and it pushes $\gamma$ to 


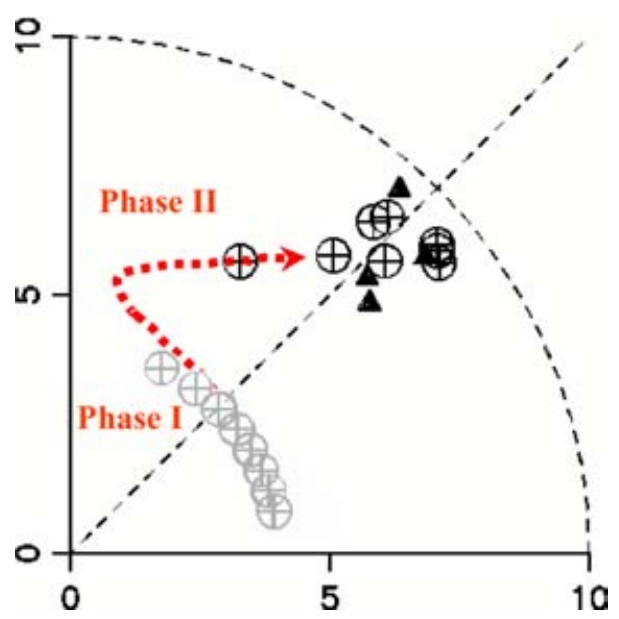

Figure 5. Period vs. obliquity polar plot depicting Slivan's prograde-rotating group. The observed asteroids are denoted by triangles, the initial state of the numerical model by gray circles and the final state after 2.5 Gy by black circles. The dashed line with an arrow shows an evolutionary path and two phases: (i) the YORP driven and (ii) the resonance capture. Adapted from Vokrouhlický et al. (2003).

$\sim 50^{\circ}, P$ to $\sim 8 \mathrm{~h}$ and also forces the spin axes to be really parallel in space. Around the time $2.5 \mathrm{~Gy}$, what is an approximate age of the Koronis family, the match of the model with the observations is perfect. Similarly, it is possible to explain the existence of the retrograde-rotating group; there is no significant spin-orbit resonance in this case and the spin axes of the retrograde-rotating asteroids are let to evolve freely toward the YORP asymptotic states.

Generally, thermal torques seem to be more important than collisions for asteroids smaller than $40 \mathrm{~km}$, because today we can still clearly see the traces of the YORP-driven evolution and the collisions have not been able to randomise the spin states during several past Gy.

\section{Delivery into unstable regions}

Various unstable populations, like meteoroids hitting the Earth, Near-Earth asteroids, or Main-Belt asteroids located inside major mean motion resonances, have dynamical lifetimes shorter than the age of the Solar System and provide a nice opportunity for dynamicists to look for sources and transport mechanisms.

\subsection{Meteorite transport from the Main Belt}

Meteorite transport from the Main Belt is the eldest application of the Yarkovsky effect (Öpik 1951; Peterson 1976; Farinella et al. 1998; Vokrouhlický \& Farinella 2000; Bottke et al. 2000). The meteorites reach the Earth in two stages: (i) a Yarkovsky-driven change of the semimajor axis spanning $\sim 10 \mathrm{My}$, and (ii) a capture in a powerful gravitational resonance, which increases eccentricity of the orbit up to 1 in a mere $\sim 1 \mathrm{My}$ (Figure 6). Approximately 1\% of meteoroids then collide with the Earth (and can be found as meteorites), but most of them fall directly to the Sun.

The main motivation for the introduction of the above Yarkovsky model were the observed cosmic ray exposure (CRE) ages of meteorites, which measure, how long time the meteorite spent in the interplanetary space as a small fragment. The model naturally explains that (i) the $\mathrm{CRE}$ ages are much longer than resonance residence times alone; 


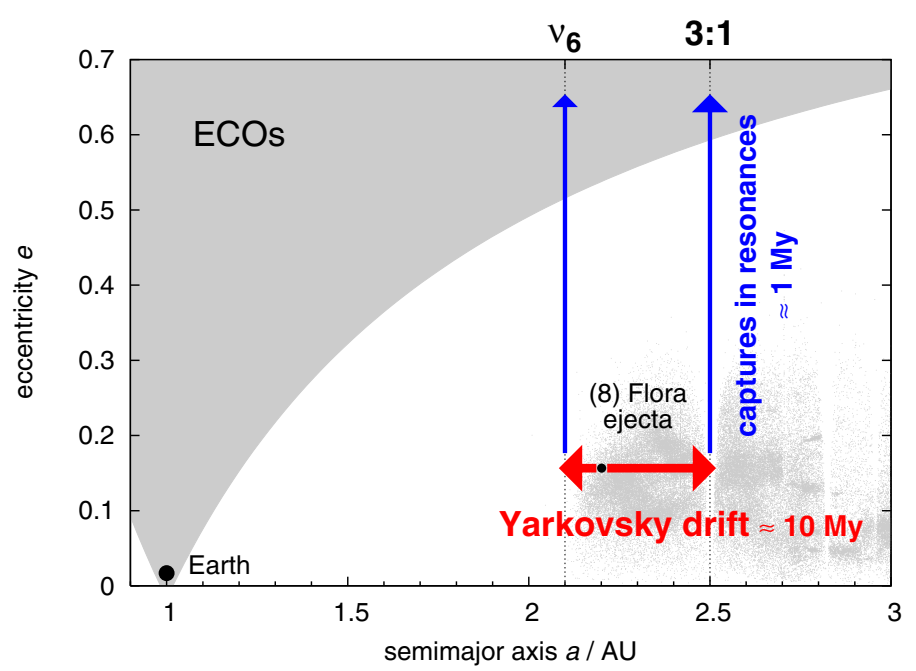

Figure 6. A schematic semimajor axis vs. eccentricity plot of the Yarkovsky-enabled model for the meteorite transport from the Main Belt. In the first stage, spanning typically $\sim 10 \mathrm{My}$, the Yarkovsky effect pushes the semimajor axes of meteoroids toward principal gravitational resonances (like $\nu_{6}$ secular resonance with Saturn and 3/1 mean motion resonance with Jupiter). In the second stage, the resonances pump the eccentricities quickly and thus in $\sim 1 \mathrm{My}$ the orbit reaches Earth-crossing space.

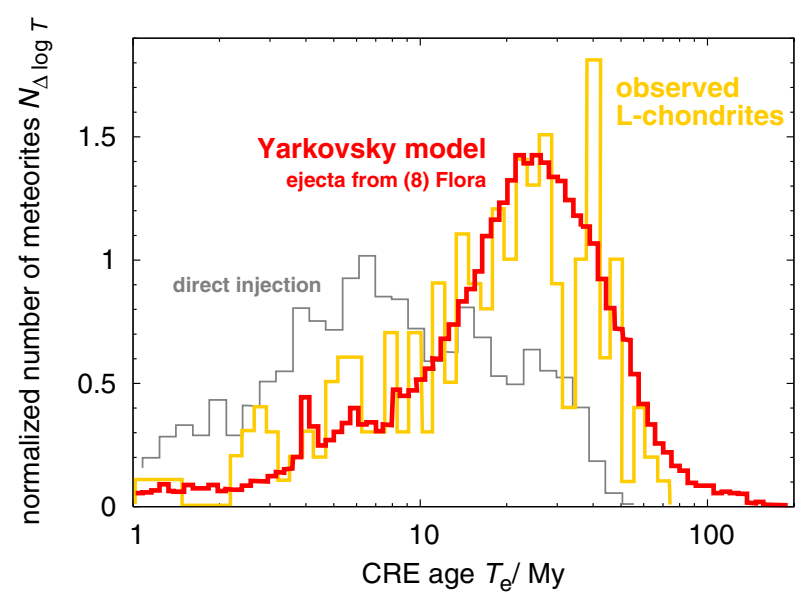

Figure 7. The observed distribution of cosmic ray exposure ages of L-chondrites (thick gray line), compared with the model distribution of Yarkovsky-driven ejecta from (8) Flora (bold line) and with an old model (thin gray line), which assumed only a direct injection of fragments into resonances. The non-random peaks on the observed CRE distribution, which were not possible to fit within a steady-state model, are most probably stochastic events, i.e., large craterings or disruptions, which produced many fragments at once. Adapted from Vokrouhlický \& Farinella (2000).

(ii) there is a strong dependence of the CRE's on the material - namely the CRE's of iron meteorites are $10 \times$ longer than of stones; (iii) the most stony meteorites have the CRE's of the order $10 \mathrm{My}$ (see Figure 7). The Yarkovsky drift is able to supply meteoroids from a wide range of parent bodies (not only from the vicinity of resonances); it is effective enough to explain the observed meteorite flux of the order $3 \times 10^{5} \mathrm{~kg} / \mathrm{y}$. Moreover, petrologic and mineralogical studies (Burbine et al. 2003) show the number 


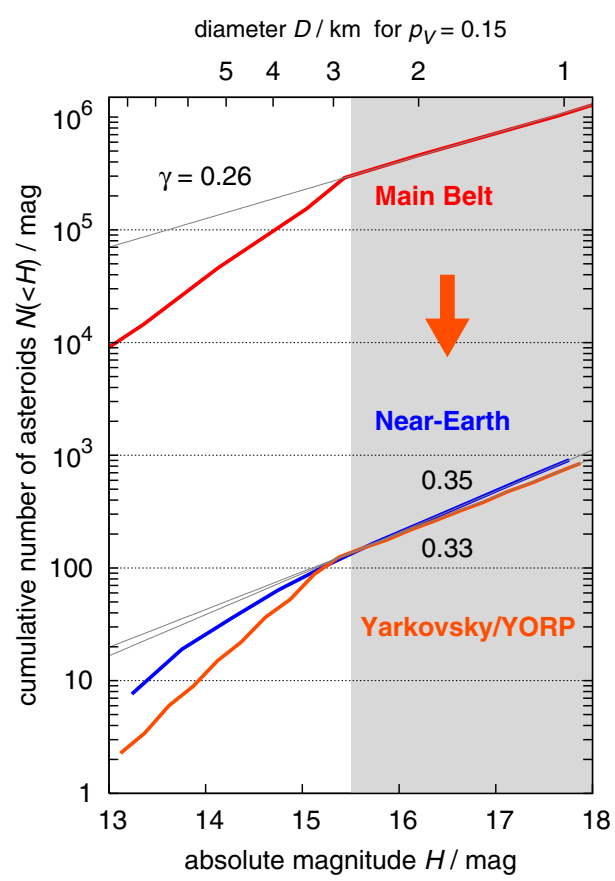

Figure 8. Cumulative distribution of absolute magnitudes $H$ for the three populations: observed Main-Belt Asteroids, observed Near-Earth Asteroids and the Yarkovsky/YORP model population, which assumes transport from the Main Belt to the near-Earth space. The slopes $\gamma$ of the distributions $\left(N(<H) \sim 10^{\gamma H}\right)$ were all fitted in the interval $H \in(15.5,18)$ mag. Adapted from Morbidelli \& Vokrouhlický (2003).

of parent bodies of iron meteorites is larger than of stones. This is because hard irons are more resistant to collisions, their total semimajor-axis drift (within the collisional lifetime) is larger and thus they can effectively sample larger volume of the Main Asteroid Belt.

\subsection{Delivery of Near-Earth Asteroids from the Main Belt}

Observations of the Near-Earth Asteroids provide two important constraints: (i) the cumulative distribution of their absolute magnitudes has a slope $\gamma=0.35(N(<H) \sim$ $10^{\gamma H}$ in the magnitude range 15.5 to 18 ; Figure 8), and (ii) their removal rate by planetary scattering is $\sim 200$ bodies larger than $1 \mathrm{~km}$ per My.

Morbidelli \& Vokrouhlický (2003) assumed the same basic scenario as for meteorites and constructed a Yarkovsky/YORP model of the transport from the Main Asteroid Belt (this source has the slope $\gamma=0.26$, again in the interval $H \in(15.5,18)$ mag). Their model yield a flux of $150-200$ bodies $\left(>1 \mathrm{~km}\right.$ ) into the main J3/1 and $\nu_{6}$ resonances (which then quickly became NEA's) and the slope of the resulting model NEA population is $\gamma=0.33$. So, the Yarkovsky/YORP effect is efficient enough to keep the current NEA population in steady state and it also explains, why the observed slope of NEA's is moderately shallower than that of MBA's.

\subsection{Resonant populations resupplied from the Main Belt}

Low-order mean motion resonances with Jupiter usually harbour small populations of objects with dynamically unstable orbits (and sometimes also stable ones). We consider here the $\mathrm{J} 7 / 3$ resonance at approximately $2.96 \mathrm{AU}$ heliocentric distance and the J2/1 


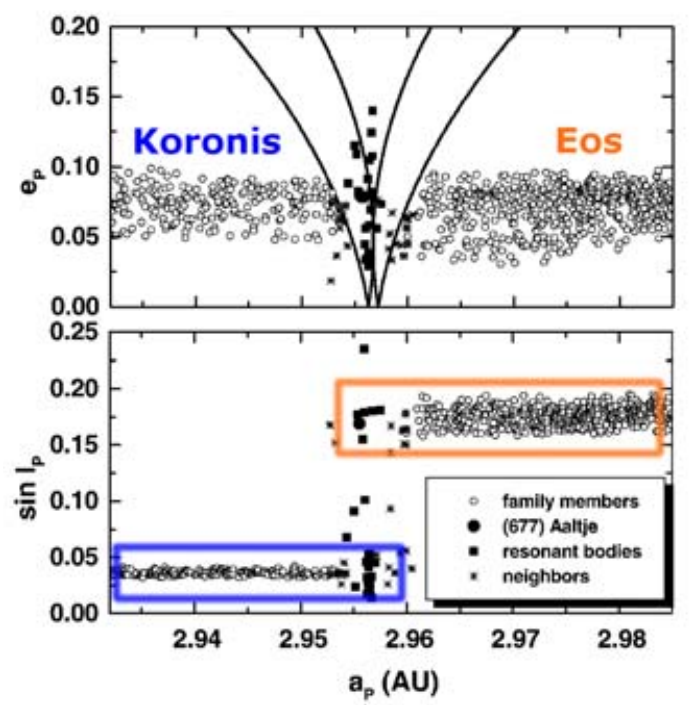

Figure 9. Proper semimajor axis vs. proper eccentricity and inclination in the surroundings of $7 / 3$ mean motion resonance with Jupiter. The resonant asteroids and two adjacent asteroid families, the Koronis and Eos, are plotted. From Tsiganis et al. (2003).

resonance at approximately $3.25 \mathrm{AU}$ as two examples, which were previously studied in some detail.

There are 22 observed unstable asteroids in the $\mathrm{J} 7 / 3$ resonance. Tsiganis et al. (2003) proved, that the Yarkovsky drift may keep the resonant population in steady state, as it pushes members of the neighbouring Koronis and Eos families towards the resonance. An independent confirmation, that the resonant bodies are truly related to the families is the observed confinement of inclinations - the mean inclinations of the two resonant groups, $2^{\circ}$ and $10^{\circ}$ respectively, correspond to the mean inclinations of the Koronis and the Eos family (Figure 9).

The J2/1 resonance harbours some 150 asteroids and 50 of them are on dynamically unstable orbits. Brož et al. (2005) simulated the evolution of neighbouring Main-Belt asteroids pushed by the Yarkovsky effect towards the J2/1 resonance. They verified this flux of Main-Belt bodies keeps the unstable resonant population in steady state. Moreover, the orbital evolutionary tracks of the Main-Belt asteroids, their dynamical lifetimes inside the $\mathrm{J} 2 / 1$ resonance and also size distribution are consistent with the actual observed unstable resonant asteroids. A few observed unstable objects, which escape from the $\mathrm{J} 2 / 1$ in less then $2 \mathrm{My}$, are most probably inactive Jupiter-Family comets.

The long-lived asteroids, confined to stable island of the J2/1 resonance, cannot be explained within the Yarkovsky model and the problem of their origin remains open.

\section{Processes shaping asteroid families}

Asteroid families are prominent clusters of asteroids, which are located close to each other in the space of proper elements $a_{p}, e_{p}$ and $\sin I_{p}$ and usually also exhibit some spectral similarities. Families are thought to be remnants of large collisions producing fragments, which then has been evolving due to the Yarkovsky/YORP effect, gravitational resonances and further secondary collisions. The primary collisions can scale from large catastrophic disruptions of parent bodies to smaller cratering events (Michel et al. 

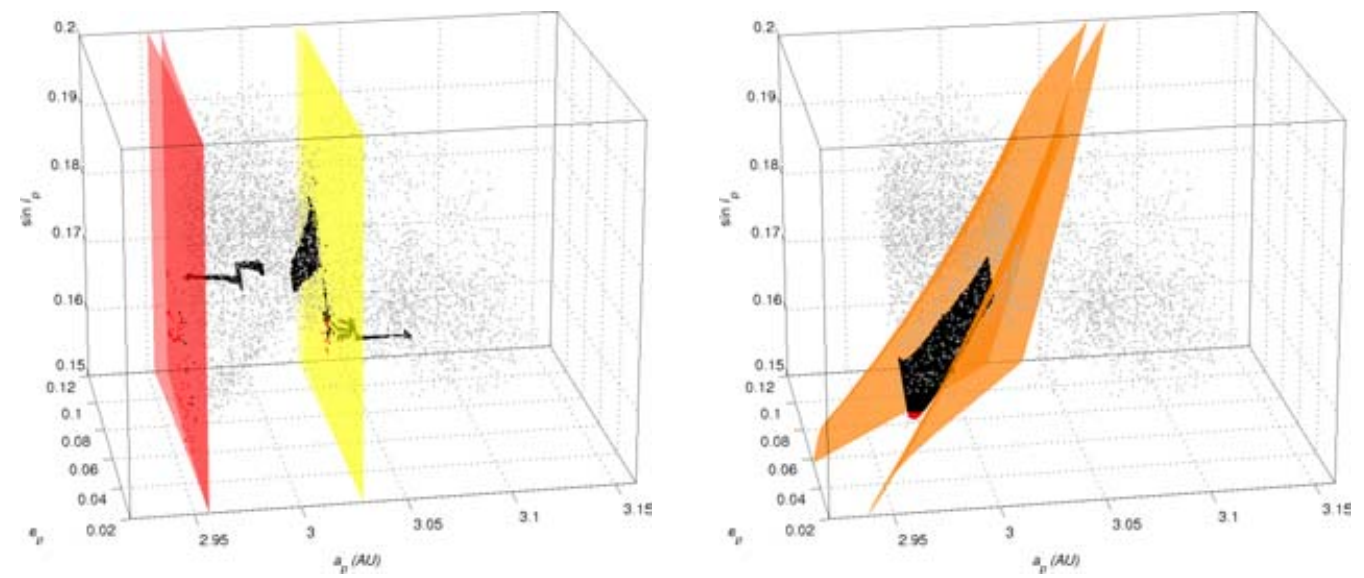

Figure 10. The Eos family in the 3-dimensional space of proper elements $a_{p}, e_{p}$ and $\sin I_{p}$. The three resonances, $\mathrm{J} 7 / 3$ and $\mathrm{J} 9 / 4$ (left) and $z_{1}$ (right) are plotted together with examples of bodies drifting by the Yarkovsky effect and interacting with these resonances. Adapted from Vokrouhlický et al. (2005a).

2001, Durda et al. 2005). Typical velocities, which fragments gain with respect to the parent body, are of the order of a few tens of $\mathrm{m} / \mathrm{s}$.

Bottke et al. (2001) and Vokrouhlický et al. (2005a) demonstrated the post-impact evolution of asteroid families using two examples: the Koronis and the Eos family. They reported three general processes, how the Yarkovsky drift together with gravitational resonances can dramatically affect the overall shape of the families, i.e., the distribution of their members in the space of proper orbital elements. We can call these processes "bracketing", "crossing" and "trapping".

At first, notice the shape of the Eos family (Figure 10): it is sharply cut at a low value of proper semimajor axis $a_{p}$, there is a evident paucity of asteroids, especially the bigger ones, at large- $a_{p}$ 's and the family is also somewhat distorted or elongated towards low- $a_{p}$, low- $e_{p}$ and low-sin $I_{p}$. These observed features nicely coincide with analytically computed borders of resonances, namely with the $7 / 3$ mean motion resonance with Jupiter at $2.955 \mathrm{AU}$, the $\mathrm{J} 9 / 4$ resonance at $3.03 \mathrm{AU}$ and the $z_{1}=g-g_{6}+s-s_{6}$ secular resonance.

We explain the observations this way: initially, just after the parent body disruption, the family was more compact; asteroids drifting due to the Yarkovsky/YORP effect towards smaller semimajor axis meet the powerful $\mathrm{J} 7 / 3$ resonance, which scatters their eccentricities and inclinations, or pumps them up to planet crossing orbits, and consequently no asteroids are visible behind. The $\mathrm{J} 7 / 3$ resonance thus brackets the Eos family (Figure 10, left).

The asteroids drifting in the opposite direction, towards larger semimajor axis, meet the weaker J9/4 resonance. Some of them are able to cross it, but the rest is scattered. This crossing explains, why there is less asteroids behind the $\mathrm{J} 9 / 4$, and why the paucity is size-dependent - the smaller asteroids drift faster and typically cross the J9/4 resonance at low eccentricity and inclination (Figure 10, left).

Many Eos-family members are trapped in the $z_{1}$ secular resonance; they drift in semimajor axis by the Yarkovsky effect and they are also forced to follow the libration centre of the resonance, which position, however, depends on all three orbital elements $a_{p}, e_{p}$ and $\sin I_{p}$. Thus, not only the semimajor axis changes, but also eccentricity and inclination and the stream of asteroids forms at small values of $a_{p}, e_{p}$ and $\sin I_{p}$, i.e., the elongated shape of the family (Figure 10, right). 


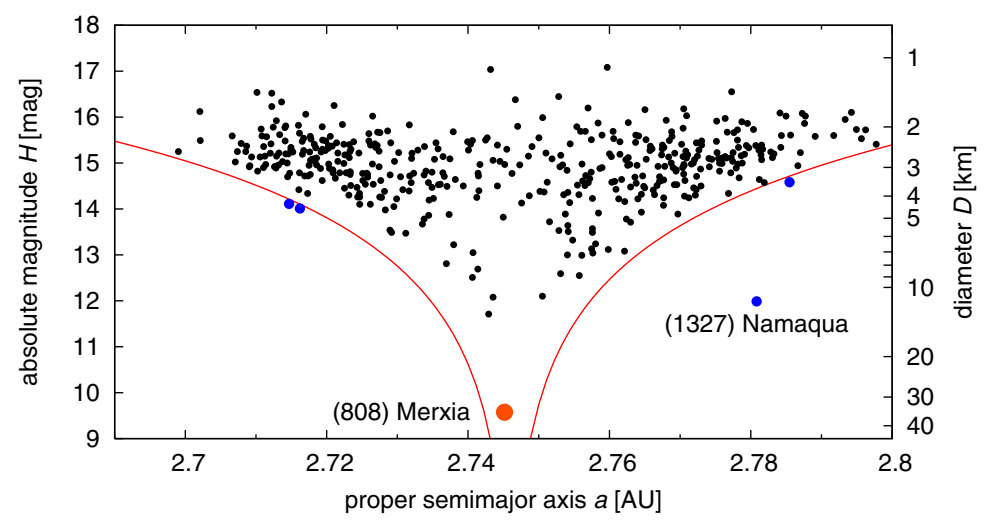

Figure 11. The Merxia family members (identified by the HCM method at the cut-off velocity $80 \mathrm{~m} / \mathrm{s}$ ) in the semimajor axis-absolute magnitude plot. The gray dots outside the ' $\mathrm{V}$ '-shape are probable interlopers.

In case of the Koronis family the situation is slightly different. This family is split in two parts, each of which has a different mean value of proper $e_{p}$ (but the same mean $\left.\sin I_{p}\right)$. Their division correlates with the position of the secular resonance $g-2 g_{5}+3 g_{6}$. A detailed study shows that, unlike in the Eos case, long-lasting captures in this resonance are not possible and drifting orbits necessarily jump over it. During this process their $e_{p}$ is always lifted by $\sim 0.025$, right the observed difference between the mean $e_{p}$ values of the two parts of the Koronis family. Because the resonance does not involve $s$-frequencies, the inclinations are not affected at all.

To conclude, if one assumes an initially compact impact-generated family (with a reasonable ejection velocity field compatible with hydrocode models), and takes into account the above evolutionary processes, it is possible to understand the currently observed extent of the family and its overall shape.

\section{1. "Eared" families and a new method of family-age determination}

The age of an asteroid family, i.e., the time of the collision which generated the family, is a very important parameter, not only for dynamical studies, but also for physical ones, space-weathering models, etc. One indication of the family age seems to be a typical ' $\mathrm{V}$ 'shape, which many families exhibit in the proper semimajor axis $a_{p}$-absolute magnitude $H$ plane; see Figure 11 for an example of the Merxia family. This shape is a natural consequence of two phenomena: (i) the initial impact, because smaller fragments (with higher $H$ 's) gain higher velocities with respect to the parent body and fall farther from the centre, and (ii) the Yarkovsky/YORP effect, because the smaller fragments drift faster in semimajor axis and subsequently move farther from the centre.

There are several outliers visible at the $\left(a_{p}, H\right)$ plot, which do not fit to this scheme. Most probably, they are interlopers, which are not related to the Merxia family. Indeed, the big asteroid (1327) Namaqua is an X-type, which is spectrally incompatible with the S-type Merxia family asteroids.

The problem is, that we do not know the initial spread, just after the impact and we cannot calculate the age simply from the current extent of the family, since the Yarkovsky drift is only responsible for an unknown part of it. Luckily, there is more information hidden in the $\left(a_{p}, H\right)$ plot - notice the depletion of small asteroids in the centre and the overdensity at extreme values of the semimajor axis. Sometimes we call this funny feature an "eared" family. Might this be a YORP effect fingerprint? The YORP effect tilts the 


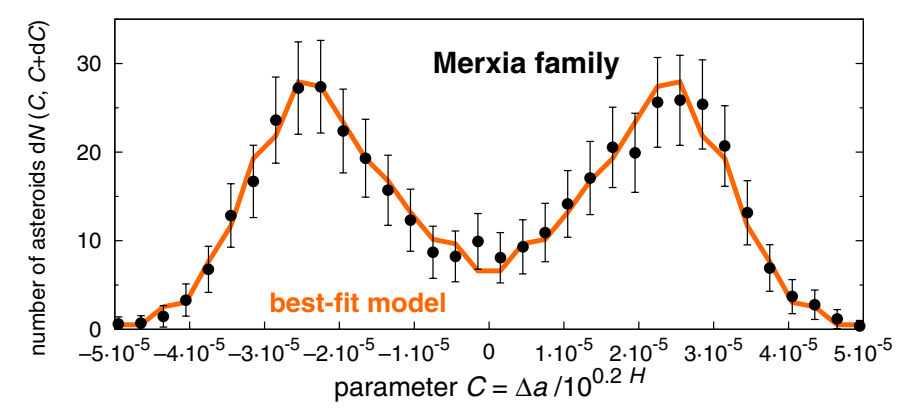

Figure 12. The distribution of the Merxia family members in the $C$-parameter and the comparison with the best fit model by Vokrouhlický et al. (2005b).

Table 2. List of asteroid families and their ages estimated by the method of Vokrouhlický et al. (2005b).

\begin{tabular}{lclr}
\hline family & age/My & family & age/My \\
\hline Agnia & $100_{-20}^{+30}$ & Erigone & $280_{-50}^{+30}$ \\
Astrid & $180_{-40}^{+80}$ & Massalia & $152_{-18}^{+18}$ \\
Eos & $1300_{-200}^{+150}$ & Merxia & $238_{+52}^{-23}$ \\
\hline
\end{tabular}

spin axes of asteroids directly up or down what enhances the Yarkovsky semimajor-axis drift and can drive the smaller asteroids towards the edges of the family. Possibly, it can allow us to resolve the ambiguity and to determine the age more precisely.

To check it, Vokrouhlický et al. (2005b) constructed a family evolution model, which accounts for: (i) an isotropic ejection of fragments (and random periods $P$ and obliquities $\gamma$ at the beginning), (ii) the Yarkovsky drift, (iii) the YORP effect, and (iv) collisional reorientations. There are four free parameters in the model: (i) the initial velocity dispersion $V$ of 5 -km fragments (for a size $D, V(D)=V \frac{5 \mathrm{~km}}{D}$ ), (ii) the YORP "strength" $c_{\text {YORP }}$ (iii) the family age $T$, and (iv) the surface thermal conductivity $K$.

They fit this model with observations using a 1-dimensional $C$-parameter, which is closely related to the semimajor axis $a_{p}$ and the absolute magnitude $H$ : $C=\Delta a_{p} / 10^{0.2 H}$, where $\Delta a_{p}$ is the distance from the family centre. The best fit for the Merxia family (Figure 12) yields the following results: the initial dispersion in semimajor axis was roughly one half of the currently observed one (what is in agreement with a statistical argument of Dell'Oro et al., 2004); the initial velocity was small $\left(V=24_{-12}^{+6} \mathrm{~m} / \mathrm{s}\right)$, what is in agreement with impact models (Michel et al. 2001); the YORP effect is important $\left(c_{\text {YORP }}=0.6_{-0.4}^{+1.4}\right) ;$ asteroids are probably covered with a low-conductivity layer $(K=$ $0.005 \mathrm{~W} / \mathrm{m} / \mathrm{K})$; and the family is of the young age $\left(T=238_{-23}^{+52} \mathrm{My}\right)$. See Table 2 for results concerning other asteroid families.

Up to now, the analysis of the Merxia family was done in the $\left(a_{p}, H\right)$ plane only. We can, however, use also information hidden in the proper eccentricity $e_{p}$ and inclination $\sin I_{p}$. The distribution of the Merxia members is clearly uneven in the $\left(a_{p}, e_{p}\right)$ plane - the spread of $e_{p}$ increases abruptly at $a_{p} \doteq 2.75$ AU. Vokrouhlický et al. (2005b) successfully explain it as a Yarkovsky transport across the three-body mean motion resonance with Jupiter and Saturn 3J-1S-1. It is actually an independent confirmation that the Yarkovsky semimajor-axis drift is calculated correctly, because the smaller spread of $e_{p}$ before the resonance is increased by the resonance crossing and then matches the observed spread of the family members behind the resonance. 
The chronology method mentioned in this section does not work for "too young" or "too old" families. The former have not had enough time to evolve by the Yarkovsky/YORP and to exhibit the "ears". The latter are much older than the typical time-scale of the YORP-driven evolution and the model does not account for the evolution of totally spun-up or spun-down asteroids.

Let us finally mention, that the freshest clusters, like Karin, Iannini or Veritas, were precisely dated by a direct backward N-body integration, which revealed a convergence of orbital perihelia and nodes corresponding to the time of the impact (Nesvorný et al. 2002, 2003; Nesvorný \& Bottke 2004; see also the review by Nesvorný et al., this volume).

\section{Conclusions and future work}

The non-gravitational forces, namely the Yarkovsky/YORP effect relevant for small asteroidal bodies in the size-range $10 \mathrm{~cm}$ to $10 \mathrm{~km}$, are now inevitable ingredients of dynamical models. Today, there is a dozen of "big" applications of the Yarkovsky/YORP models; we mentioned some of them in this brief review.

The precise measurement of Golevka's non-gravitational drift was only a first step. Within the next decade, we expect a dozen of similar Yarkovsky detections by precise radar astrometry (Vokrouhlický et al. 2005c, 2005d) or future optical astrometry with GAIA.

Yarkovsky semimajor-axis drift of the order $\sim 10 \mathrm{~km}$ per 10 years becomes crucial for an accurate orbit determination and even for estimates of an impact hazard (Giorgini et al. 2002). Especially, when the calculation of an impact probability depends on the fact, if the asteroid misses or hits a phase-space "keyhole", which is much smaller then the diameter of the Earth.

Further step forward might be a thorough combination of dynamical models with infrared observations of NEA's and their thermophysical models (Delbó et al. 2003) they supply independent constraints (with different correlations) on Yarkovsky/YORPrelated parameters, like the thermal conductivity.

We can await the first direct detection of the YORP effect in the forthcoming years, either from ground-based photometric measurements and corresponding lightcurve modelling, or from the space-borne mission Hayabusa, which now orbits the asteroid (25143) Itokawa (e.g., Vokrouhlický et al. 2004).

The dynamical studies of asteroid families provide also predictions of physical properties and rotational states of individual asteroids, which can serve as good opportunities for further observational tests (similar to Vokrouhlický et al. (2005e) who photometrically observed (2953) Vysheslavia and confirmed its retrograde rotation predicted by Vokrouhlický et al. (2001)). For example, the small members of the families with intermediate ages (discussed in Section 5.1) should exhibit preferential values of obliquities due to the YORP torque and Yarkovsky drift: the asteroids located far from the family-centre at lower/larger values of semimajor axis should have retrograde/prograde rotations. The most suitable families for such survey seem to be the Massalia or the Erigone, located in the inner Main Belt, what makes them more easily observable.

An appealing project would be to determine systematically the ages of all asteroid families, including large and old ones. However, we have to face several obstacles: (i) we still lack the direct measurements of basic physical parameters (albedos, masses, shapes, spectra) for most family members and we cannot expect the situation dramatically improves in the next few years; (ii) a modelling of several subsequent YORP cycles have not been developed yet. 
There is already a number of examples, how the YORP torque affects rotational states of asteroids (we discussed some in Sections 3 and 5.1). Moreover, there are further indications: (i) the distribution of rotational periods of all $\sim 1500$ asteroids, we have lightcurves for, reveals an excess of very slow and very fast rotators (Pravec \& Harris 2000); (ii) small NEA's have a non-Maxwellian distribution of periods; and (iii) there seems to exist a preference of retrograde-rotating asteroids among NEA's (La Spina et al. 2004), what is in concert with the positions of Main-Belt escape routes, fed by the obliquity-dependent Yarkovsky drift. A detailed model for a long-term YORP-driven period and obliquity evolution, concerning the entire Main-Belt and NEA's, does not exist yet. Also a possible YORP origin of binaries created by asteroid fission have not been studied in detail.

\section{Acknowledgements}

The work of DV, DČ and MB was supported by the Grant Agency of the Czech Republic by grant 205/05/2737.

\section{References}

Bottke, W.F., Rubincam, D.P., \& Burns, J.A. 2000, Icarus 145, 301

Bottke, W.F., Vokrouhlický, D., Brož, M., Nesvorný, D., \& Morbidelli, A. 2001, Science 294, 1693

Bottke, W.F., Vokrouhlický, D., Rubincam, D.P., \& Brož, M. 2002, in: W.F. Bottke, A. Cellino, P. Paolicchi \& R.P. Binzel (eds.), Asteroids III (Tucson: The University of Arizona Press), p. 395

Brož, M., Vokrouhlický, D., Roig, F., Nesvorný, D., Bottke, W.F., \& Morbidelli, A. 2005, Mon. Not. R. Astron. Soc. 359, 1437

Burbine, T.H., McCoy, T.J., Meibom, A., Gladman, B., \& Keil, K. 2003, in: W.F. Bottke, A. Cellino, P. Paolicchi \& R.P. Binzel (eds.), Asteroids III (Tucson: The University of Arizona Press), p. 653

Chesley, S.R., Ostro, S.J., Vokrouhlický, D., Čapek, D., Giorgini, J.D., Nolan, M.C., Margot, J.-L., Hine, A.A., Benner, L.A.M., \& Chamberlin, A.B. 2003, Science 302, 1739

Čapek, D. \& Vokrouhlický, D. 2005, Icarus, to be submitted

Delbó, M., Harris, A.W., Binzel, R.P., Pravec, P., \& Davies, J.K. 2003, Icarus 166, 116

Dell'Oro, A., Bigongiari, G., Paolicchi, P., \& Cellino, A. 2004, Icarus 169, 341

Durda, D.D., Bottke, W.F., Nesvorný, D., Asphaug, E., \& Richardson, D.C. 2005, Icarus, submitted

Farinella, P., Vokrouhlický, D., \& Hartmann, W.K. 1998, Icarus 132, 378

Giorgini, J.D., Ostro, S.J., Benner, L.A.M., Chodas, P.W., Chesley, S.R., Hudson, R.S., Nolan, M.C., Klemola, A.R., Standish, E.M., Jurgens, R.F., Rose, R., Chamberlin, A.B., Yeomans, D.K., \& Margot, J.-L. 2002, Science 296, 132

La Spina, A., Paolicchi, P., Kryszczynska, A., \& Pravec, P. 2004, Nature 428, 400

Michel, P., Benz, W., Tanga, P., \& Richardson, D.C. 2001, Science 294, 1696

Morbidelli, A. \& Vokrouhlický, D. 2003, Icarus 163, 120

Nesvorný, D., Bottke, W.F., Dones, L., \& Levison, H.F. 2002, Nature 417, 720

Nesvorný, D., Bottke, W.F., Levison, H.F., \& Dones, L. 2002, Ap.J. 591, 486

Nesvorný, D. \& Bottke, W.F. 2004, Icarus 170, 324

Öpik, E.J. 1951, Proc. R. Irish Acad. 54, 165

Peterson, C. 1976, Icarus 29, 91

Pravec, P. \& Harris, A.W. 2000, Icarus 148, 12

Rubincam, D.P. 1995, J. Geophys. Res. 100, 1585

Rubincam, D.P. 2000, Icarus 148, 2

Slivan, S.M. 2002, Nature 419, 49

Slivan, S.M., Binzel, R.P., Crespo da Silva, L.D., Kaasalainen, M., Lyndaker, M.M., \& Krčo, M. 2003, Icarus 162, 285

Tsiganis, K., Varvoglis, H., \& Morbidelli, A. 2003, Icarus 166, 131 
Vokrouhlický, D. 1998, Astron. Astrophys. 335, 1093

Vokrouhlický, D. 1999, Astron. Astrophys. 344, 362

Vokrouhlický, D., Brož, M., Farinella, P., \& Knežević, Z. 2001, Icarus 150, 78

Vokrouhlický, D. \& Čapek, D. 2002, Icarus 159, 449

Vokrouhlický, D. \& Farinella, P. 2000, Nature 407, 606

Vokrouhlický, D., Milani, A., \& Chesley, S.R. 2000, Icarus 148, 118

Vokrouhlický, D., Nesvorný, D., \& Bottke, W.F. 2003, Nature 425, 147

Vokrouhlický, D., Čapek, D., Kaasalainen, M., \& Ostro, S.J. 2004, Astron. Astrophys. 414, L21

Vokrouhlický, D., Brož, M., Morbidelli, A., Bottke, W.F., Nesvorný, D., Lazzaro, D., \& Rivkin, A.S. 2005a, Icarus, in press

Vokrouhlický, D., Brož, M., Bottke, W.F., Nesvorný, D., \& Morbidelli, A. 2005b, Icarus, submitted

Vokrouhlický, D., Čapek, D., Chesley, S.R., \& Ostro, S.J. 2005c, Icarus, 173, 176

Vokrouhlický, D., Čapek, D., Chesley, S.R., \& Ostro, S.J. 2005d, Icarus, in press

Vokrouhlický, D., Brož, M., Michałowski, T., Slivan, S.M., Colas F., Šarounová, L., \& Velichko, F. 2005e, Icarus, in press 